\title{
Microcalcificaciones y focos ecogénicos puntiformes, ¿qué tan bien sabemos lo que estamos viendo?
}

\author{
Carolina Herrera $A^{1,3}$, Claudio Lagos $C^{3}$, Antonieta Solar $G^{2,3}$, Yobani Díaz $C^{2,3}$, Vanessa Garzón $D^{2,3}$, Francisco \\ Cruz $\mathrm{O}^{1,3}$. \\ 1. Departamento de Radiología, Hospital Clínico Universidad Católica de Chile. Santiago, Chile. \\ 2. Departamento de Anatomía Patológica, Hospital Clínico Universidad Católica de Chile. Santiago, Chile. \\ 3. Facultad de Medicina, Pontificia Universidad Católica de Chile. Santiago, Chile.
}

\section{Microcalcifications and punctate echogenic foci, how well do we know what we are seeing?}

\begin{abstract}
The presence of microcalcifications in thyroid nodules is a very specific sign of malignancy, as it corresponds to Psammoma bodies. There are not enough studies that demonstrate a correlation between their histological presence and their actual ultrasound appearance. Materials and Methods: All nodules larger than $3 \mathrm{~cm}$ punctured at the Universidad Católica Clinical Hospital between 2010-2015 were selected, and the sonographic appearance was classified according to the presence of 3 types of echogenic foci according to a stricter definition than usual. The above was correlated with findings in biopsies. Results: 44 nodules corresponded to papillary thyroid cancer. There was a statistically significant relationship between a new ultrasound definition of the microcalcifications (punctate echogenic foci) and the histological presence of psamomma bodies. Discussion: There would be a good correlation between a stricter definition and the actual presence of microcalcifications in histology, improving the high rate of over diagnosis recently noticed by some authors.
\end{abstract}

Keywords: Thyroid, Ultrasound, Microcalcifications, Psamomma bodies.

Resumen. La presencia de microcalcificaciones en nódulos tiroideos es un signo muy específico de malignidad, al corresponder a cuerpos de Psammoma. No existen suficientes estudios que demuestren una correlación entre su presencia histológica y su aspecto ecográfico real.

Materiales y métodos: Se seleccionaron todos los nódulos con tamaño mayor a $3 \mathrm{~cm}$ puncionados en el Hospital Clínico Universidad Católica entre los años 2010-2015 y se clasificó el aspecto ecográfico según la presencia de 3 tipos de focos ecogénicos con una definición más estricta a lo usual. Se correlacionó lo anterior con hallazgos en biopsias. Resultados: 44 nódulos correspondieron a cáncer papilar de tiroides. Hubo relación estadísticamente significativa entre una nueva definición ecográfica de las microcalcificaciones (focos ecogénicos puntiformes) y la presencia histológica de cuerpos de psamomma.

Discusión: Habría una buena correlación entre una definición más estricta y la presencia real de microcalcificaciones en histología, mejorando la alta tasa de sobrediagnóstico advertido recientemente por algunos autores.

Palabras clave: Tiroides, Ultrasonido, Microcalcificaciones, Cuerpos de psamomma.

Herrera C., et al. Microcalcificaciones y focos ecogénicos puntiformes, ¿qué tan bien sabemos lo que estamos viendo? Rev Chil Radiol 2017; 23(4): 143-150.

Correspondencia: Claudio Lagos C. / crlagos@uc.cl.

Trabajo ganador del primer premio en trabajos libres del Congreso Chileno de Radiología 2017.

\section{Introducción}

En ecografía, las microcalcificaciones en nódulos tiroideos y su relevancia para el diagnóstico ha sido ampliamente estudiada, existiendo una asociación fuerte entre su presencia y el riesgo de malignidad, en especial, en relación al cáncer papilar de tiroides $(\mathrm{CPT})^{1,2}$.

Desde un punto de vista anatomopatológico, se considera que representan la presencia de cuerpos de psammoma (CPs) (Figura 1) ${ }^{3}$. 
Éstos corresponden a estructuras papilares, que luego de un proceso de necrosis evolucionan a una calcificación distrófica con una morfología laminada y concéntrica. Comúnmente son observadas en el CPT, siendo reportadas rara vez en otras lesiones neoplásicas y no neoplásicas ${ }^{4}$. No obstante, el aspecto ecográfico de las microcalcificaciones puede confundirse con focos ecogénicos de otra naturaleza, existiendo una alta variabilidad interobservador en el diagnóstico de las mismas, tema que no ha sido suficientemente estudiado, pero del que empíricamente se tiene la impresión de una tendencia marcada al sobrediagnóstico ${ }^{5}$.

Entre los múltiples factores que pueden explicar el problema, uno de los más importantes es la ausencia de evidencia que demuestre una correlación entre lo clásicamente aceptado como microcalcificaciones en ecografía y la presencia real de CPs.

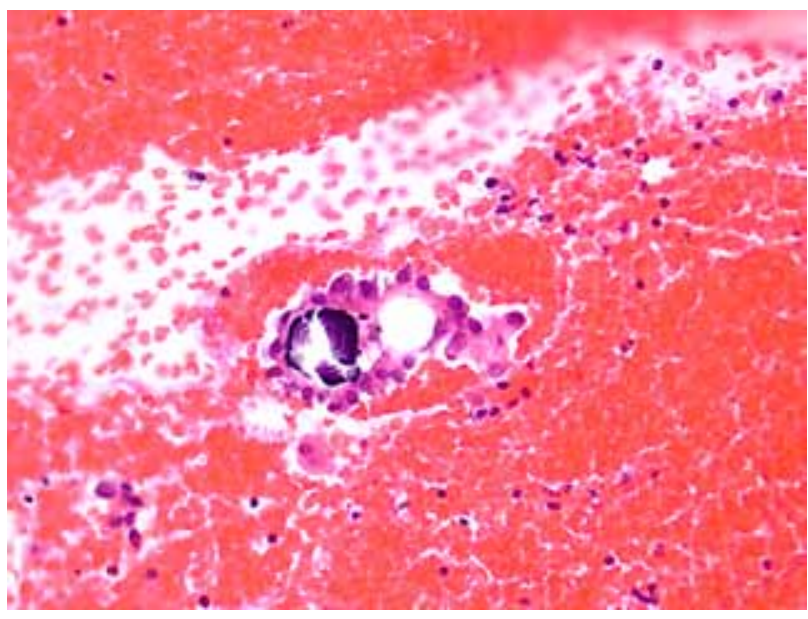

Figura 1. Se observa al centro de la imagen una microcalcificación fragmentada, correspondiente a un cuerpo de Psamomma.

\section{Objetivo}

Proponer una clasificación de los focos ecogénicos visualizados por ecografía en nódulos tiroideos, que en general se consideran microcalcificaciones, definiendo en forma más precisa cuales son los que se asocian con mayor probabilidad a CPs, haciendo la correlación eco/cito-histológica de microcalcificaciones en nódulos de pacientes con cáncer papilar de tiroides, para establecer la precisión diagnóstica del método ecográfico y el grado de asociación estadística entre las microcalcificaciones, ahora con una definición más estricta (focos ecogénicos tipo 1) y la presencia real de CPs en el examen histopatológico.

\section{Materiales y métodos}

Estudio retrospectivo enmarcado dentro de otro estudio de mayor envergadura en relación a las características ecográficas de nódulos tiroideos con tamaño mayor a $3 \mathrm{~cm}$. Se revisaron ecografías de punciones con sus informes, resultado de citologías (PAF) y biopsias de nódulos $>3 \mathrm{~cm}$ entre enero de 2010 y diciembre de 2015 disponibles en el sistema PACS-RIS ${ }^{\circledR}$ de la red de salud UC-Christus.

Se incluyó para el análisis estadístico a 44 pacientes con CPT. La muestra seleccionada correspondía a pacientes con CPT en PAF con posterior confirmación con biopsia quirúrgica o seguimiento clínico concordante en pacientes no candidatos a resección quirúrgica.

Con respecto a la búsqueda de CPs, se revisaron en detalle los informes en anatomía patológica de las PAF de los 44 nódulos, y se re-analizaron las placas de biopsias quirúrgicas llevadas a cabo en nuestra institución $(n=16)$. La revisión de placas fue llevada a cabo por dos operadores entrenados con 2-3 años de experiencia, y certificados por una anatomopatóloga con 15 años de experiencia en patología tiroidea.

La recopilación de datos en relación a los informes ecográficos fue realizada por dos operadores con 1,5 y 3 años de experiencia en el tema, seleccionados y entrenados por un radiólogo con 37 años de experiencia en ecografía de tiroides, en la clasificación y caracterización de nódulos tiroideos. Se revisaron 4.462 ecografías de punciones entre las fechas descritas y se seleccionaron todos los nódulos con un tamaño $>3 \mathrm{~cm}(\mathrm{n}=455)$. Se registraron las características ecográficas y la concordancia con los informes, siendo revisados por el operador experimentado todos los casos con discordancia informe-imagen o aquellos en los que no se encontró informe y los operadores entrenados consideraron que requerían revisión del experto para la determinación final de los caracteres del nódulo. Como control de calidad en relación al presente trabajo, se solicitó la revisión ciega por parte del experto de los 44 casos con CPT para los focos ecogénicos en estudio.

\section{Clasificación de los distintos focos ecogénicos}

Con respecto a la clasificación ecográfica empleada para los distintos tipos de focos, se utilizó como base el último consenso para la clasificación ecográfica de nódulos tiroideos propuesto por el Colegio Americano de Radiología $(A C R)^{6}$ y se realizó una adaptación en función de resolver los conflictos que ella plantea, llegando a las siguientes definiciones:

- Tipo 1: Focos ecogénicos puntiformes (FEP) (equivalentes a microcalcificaciones según dicho consenso), definidos como "focos ecogénicos puntiformes con un tamaño menor a $1 \mathrm{~mm}$, sin sombra acústica". A lo anterior propuesto por el $A C R$, agregamos algunas características que ayudan al diagnóstico: cuando están de preferencia agrupados en el espesor del tejido sólido; se encuentran alineadas en la periferia del nódulo y cuando aparecen con un patrón tipo "sal y pi- 
mienta". Excepcionalmente, si un conglomerado de microcalcificaciones se agrupa focalmente puede generar una sombra acústica y esto no las descarta (Figuras 2, 3, 4 y 5).

- Tipo 2: Focos ecogénicos inespecíficos (FEI) definidos como focos de algo mayor tamaño (1-2 mm), de mayor ecogenicidad que los FEP, sin artefacto en cola de cometa $0<$ de $1 \mathrm{~mm}$, más bien aislados y en relación a microquistes (Figuras 6 y 7 ).

- Tipo 3: Focos de coloide espeso definidos por la presencia de artefacto en "cola de cometa" mayor a $1 \mathrm{~mm}$ y el resto de las características ecográficas similar a los FEI (Figuras 8 y 9).

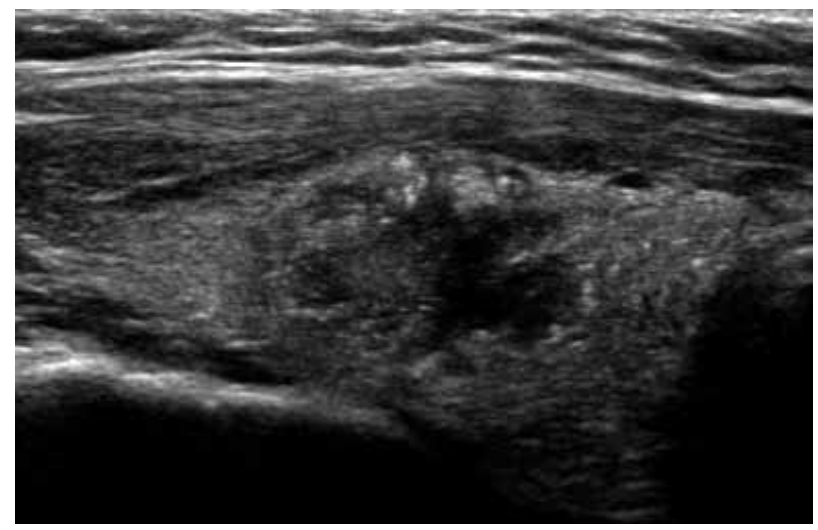

Figura 2. Focos ecogénicos puntiformes (FEP), según lo planteado por nuestra definición.

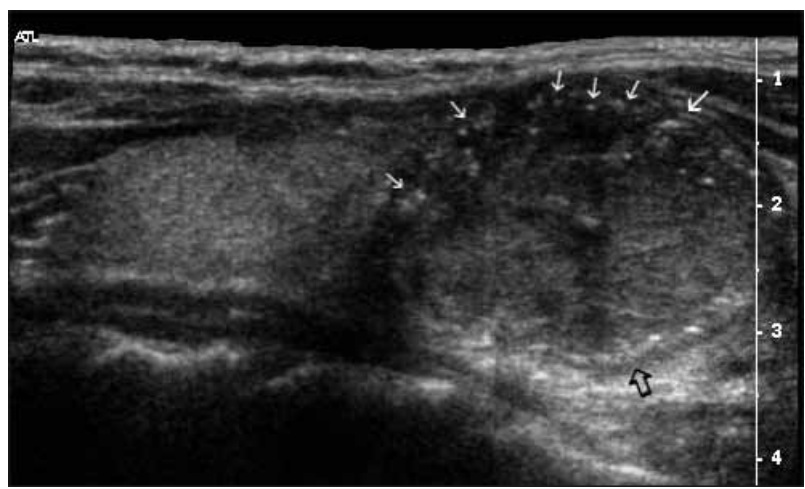

Figura 3. Focos ecogénicos tipo 1, en la periferia del nódulo.

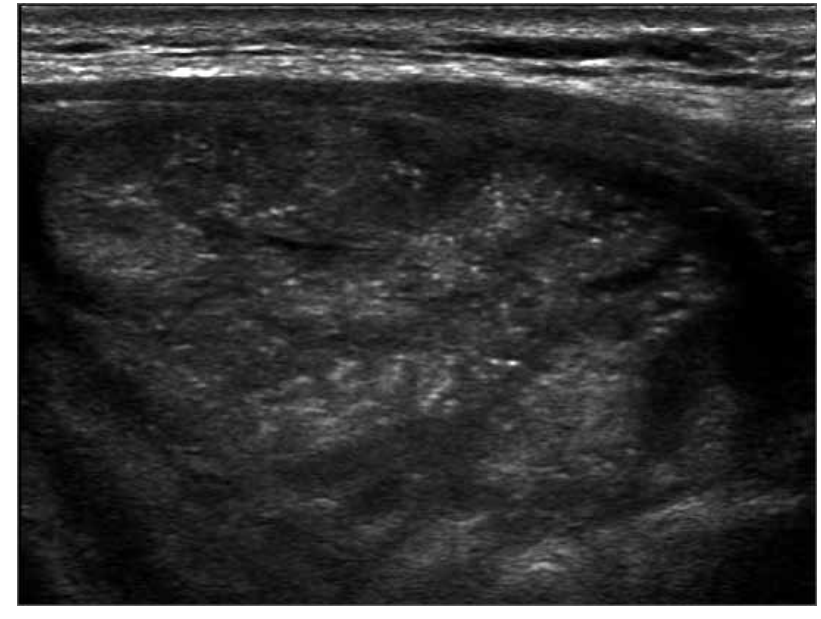

Figura 4. Focos ecogénicos puntiformes en patrón tipo sal y pimienta.

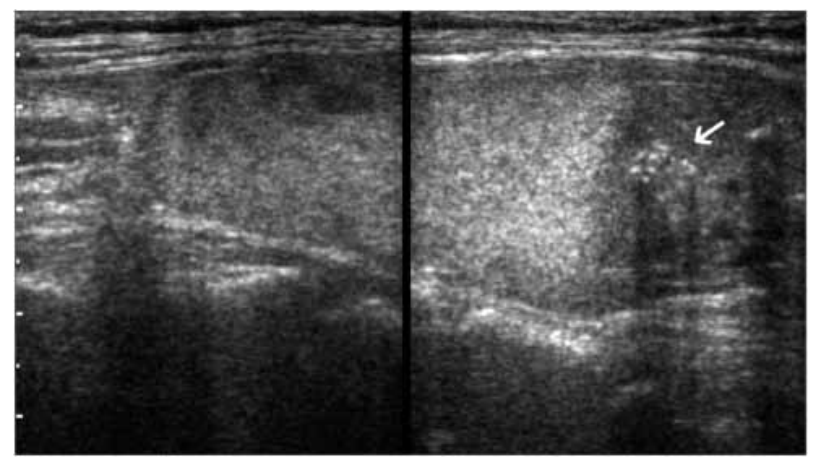

Figura 5. Conglomerado de focos ecogénicos con sombra acústica posterior al grupo.

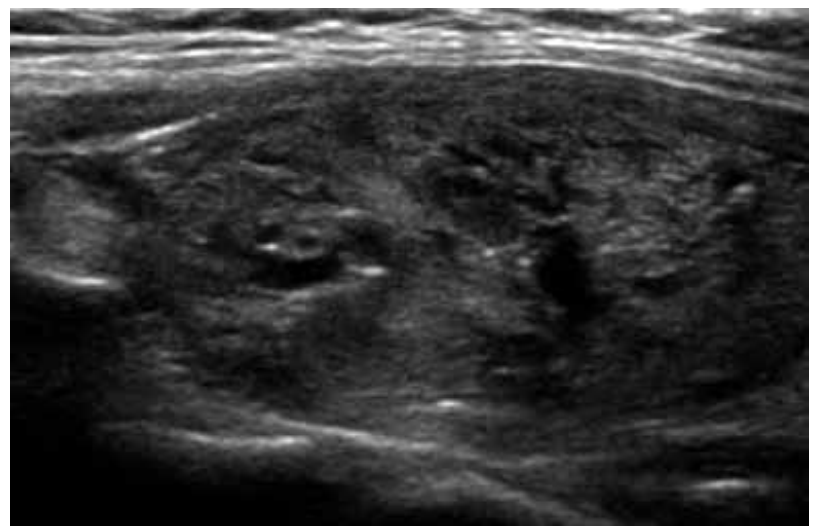

Figura 6. Focos ecogénicos inespecíficos (FEI), se observan distribuidos de forma aleatoria, con ecogenicidad mayor a la de FEP. 


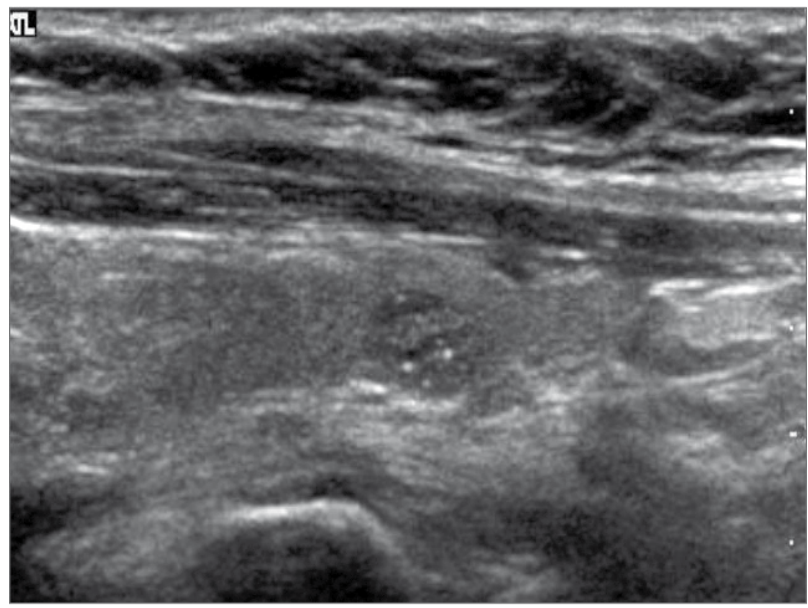

Figura 7. Focos ecogénicos inespecíficos (FEI).

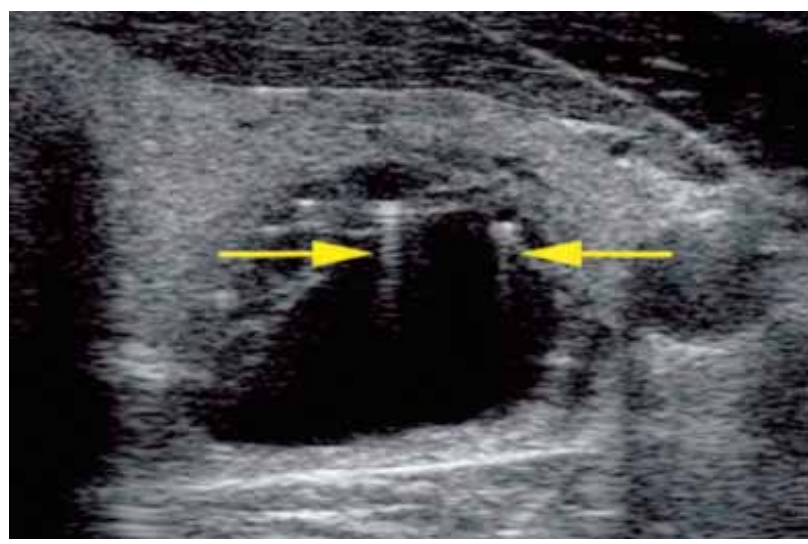

Figura 8. Focos de coloide espeso. Con las flechas amarillas se indica el artefacto en cola de cometa $>1 \mathrm{~mm}$.

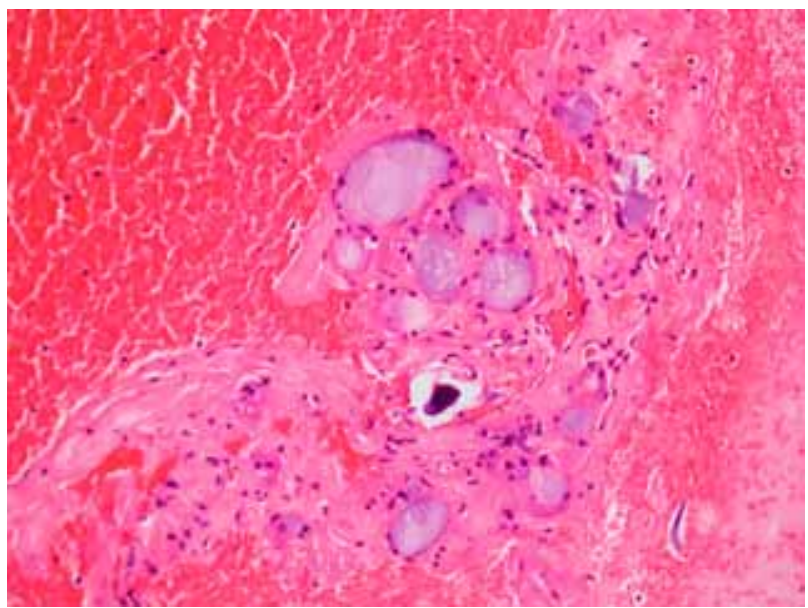

Figura 9. Aspecto microscópico de focos de coloide intranodular.

\section{Resultados}

Dentro del grupo general del trabajo $(n=455)$ se obtuvo inicialmente el total de CPT, correspondiente a 44 nódulos $(9,67 \%)$ con tamaño $>3 \mathrm{~cm}$. Se obtuvo la distribución de frecuencias de los distintos focos ecogénicos según la definición adoptada [IC 95\%] para el grupo general, como se puede apreciar en la tabla 1.

Tabla 1. Distribución de los distintos focos ecogénicos estudiados en el grupo general de nódulos $>3 \mathrm{~cm}(\mathrm{n}=455)$.

\begin{tabular}{|l|c|r|r|}
\cline { 2 - 4 } & Frecuencia & \multicolumn{1}{c|}{$\%$} & [IC 95\%] \\
\hline Sin focos & 275 & 60,43 & {$[55,8-65,1]$} \\
FEP & 32 & 7,03 & {$[4,8-9,5]$} \\
FEI & 122 & 26,81 & {$[22,9-31,2]$} \\
Coloide & 26 & 5,71 & {$[3,7-7,9]$} \\
\hline
\end{tabular}

Se calcularon los grados de significancia y asociación estadística entre varias combinaciones de focos ecogénicos y malignidad o $\mathrm{CPT}$, según lo mostrado en las tablas 2 y 3 . Se calculó también la asociación entre focos de coloide espeso y benignidad para comparar con la literatura revisada, obteniéndose una diferencia estadísticamente no significativa con el grupo control $\left(X^{2} 1,775 ; p=0,183\right)$ y un coeficiente de asociación ( $\mathrm{V}$ de Cramer) insuficiente $(0,062)$.

Tabla 2. Coeficientes de significancia y asociación estadística entre focos ecogénicos y malignidad.

\begin{tabular}{|l|c|c|c|}
\cline { 2 - 4 } & Chi-cuadrado & $\mathbf{p}$ & V de Cramer \\
\hline FEP & 113,16 & $<0,001$ & 0,499 \\
FEI+FEP & 20,589 & $<0,001$ & 0,213 \\
FEI & 1,16 & 0,196 & 0,061 \\
Coloide & 1,83 & 0,176 & 0,063 \\
\hline
\end{tabular}

Tabla 3. Coeficientes de significancia y asociación estadística entre focos ecogénicos y CPT.

\begin{tabular}{|l|c|c|c|}
\cline { 2 - 4 } & Chi-cuadrado & $\mathbf{p}$ & v de Cramer \\
\hline FEP & 119,97 & $<0,001$ & 0,514 \\
FEI+FEP & 12,774 & $<0,001$ & 0,168 \\
FEI & 6,27 & $<0,05$ & 0,117 \\
Coloide & 3,03 & 0,082 & 0,082 \\
\hline
\end{tabular}


En el grupo específico de nódulos con CPT se obtuvieron: 14 nódulos sin focos; 25 con focos tipo 1 (FEP); 5 con focos tipo 2 (FEl); ninguno con focos de coloide espeso. En cuanto a la distribución porcentual de los focos ecogénicos, hubo una diferencia estadísticamente significativa $(p<0,05)$ en comparación con el grupo general, observándose un aumento importante en FEP y una disminución marcada de FEl y focos de coloide espeso en el grupo CPT, como se observa en la figura 10. Como información anexa relevante relacionada con la detección y diferenciación correcta de los distintos focos ecogénicos, no hubo nódulos quísticos y sólo 2/44 eran hiperecogénicos.

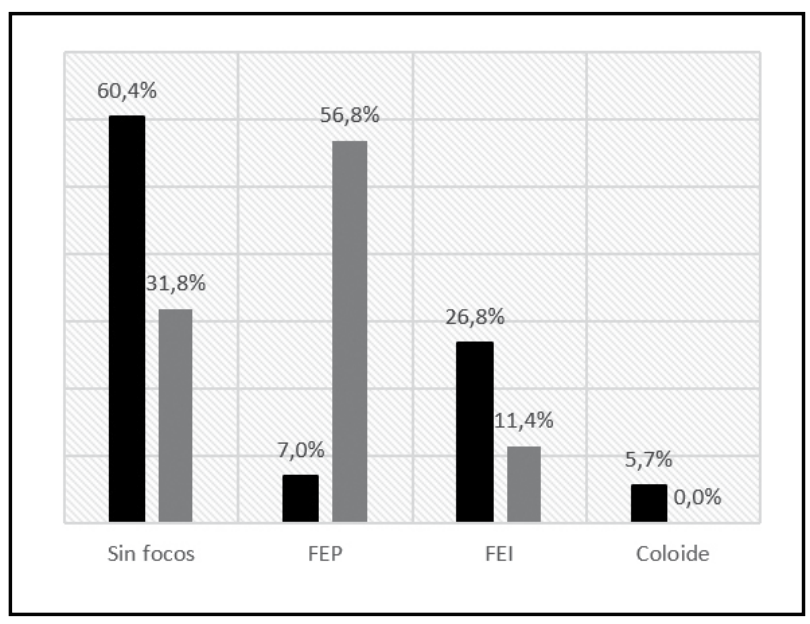

Figura 10. Distribución porcentual comparativa entre la presencia de los distintos focos ecogénicos en el grupo general (negro) vs el grupo CPT (gris).

Con respecto a la presencia efectiva de CPs en histología, se encontró que en total 14 nódulos tiroideos con CPT tenían presentes tal tipo de calcificación. De los CPs encontrados, sólo 9 fueron detectados en biopsia quirúrgica; uno detectado en biopsia y blockcelular; 4 detectados sólo en block-celular, aunque en estos últimos 4 casos no fue posible acceder a las placas de biopsias quirúrgicas. De éstos, 13 nódulos $(92,86 \%)$ fueron correctamente clasificados en ecografía (FEP); hubo un falso negativo $(7,14 \%$; se clasificó como sin focos ecogénicos); no hubo CPs en nódulos con FEl.

Para evaluar el rendimiento diagnóstico del ultrasonido para detectar correctamente CPs, se usó como Gold Standard de diagnóstico los resultados de la cito-histología, obteniéndose la sensibilidad, especificidad, VPP y VPN para nuestras definiciones con los resultados que se indican en la tabla 4 . Se puede apreciar que tanto sensibilidad, especificidad y VPN muestran resultados positivos, no obstante, se obtuvo un VPP pobre.
Tabla 4. Valoración diagnóstica de nuestra definición de FEP para PBs (microcalcificaciones reales).

$\begin{array}{ll}\text { Sensibilidad } & 92 \% \\ \text { Especificidad } & 97 \% \\ \text { VPP } & 40 \% \\ \text { VPN } & 99 \%\end{array}$

\section{Discusión}

En ecografía, el hallazgo de microcalcificaciones en un nódulo tiroideo es un signo de alta sospecha de cáncer tiroideo, fundamentalmente $\mathrm{CPT}^{1,2}$, la variedad más frecuente de cáncer tiroideo ${ }^{12}$, especialmente si se asocian a composición solida e hipoecogenicidad. Su presencia casi invariablemente va a ser motivo para indicar una PAF que por el momento es la mejor manera de avanzar en el diagnóstico. Sin embargo, la mayor parte de los focos ecogénicos no corresponden a microcalcificaciones y son muy similares entre sí, siendo necesario precisar qué características tienen aquellos que más probablemente corresponderán a microcalcificaciones, con CPs en su correlato histopatológico.

No hay consenso en la literatura con respecto a la nomenclatura de los distintos focos ecogénicos, ni a la definición pictográfica precisa de cada uno de ellos $^{6-11}$. Recientemente, un estudio publicado por un grupo con vasta experiencia en patología tiroidea ${ }^{5}$, planteó por primera vez un nuevo enfoque para resolver algunas de las interrogantes en torno al tema, evaluando el grado de asociación entre cinco distintos tipos de focos ecogénicos propuestos y el riesgo de malignidad $^{5}$. En dicho estudio se encontró una alta proporción de nódulos con focos ecogénicos, no habiendo mayor porcentaje de malignidad en comparación con el grupo sin focos ecogénicos (nódulos con focos ecogénicos; $14.9 \%$; nódulos sin focos ecogénicos, $12.2 \% ; p=0.31$ ). Sin embargo, al separar los focos con artefacto en cola de cometa mayor a $1 \mathrm{~mm}$ de los restantes tipos de focos, se vio una asociación muy fuerte con benignidad, a diferencia del grupo restante que tomado como conjunto mostraba una mayor tasa de neoplasias. Aun así, lo definido en ese estudio como "focos ecogénicos puntiformes" (equivalente a lo que la mayoría de otros trabajos llama "microcalcificaciones) mostró un aumento discreto de riesgo, observándose la presencia de dichos elementos en el $89,4 \%$ de todos los nódulos con focos ecogénicos. De estos últimos, un $84,4 \%$ correspondían a nódulos benignos. En consecuencia, se plantea un error serio en la nomenclatura utilizada hasta el día de hoy al utilizar el término microcalcificaciones con tal grado de laxitud, ya que no existe razón alguna para 
pensar que se pudiesen encontrar CPs (verdaderas microcalcificaciones) en un porcentaje tan alto de nódulos ${ }^{14}$, que además usualmente son coloideos ${ }^{5}$.

De ahí la iniciativa de proponer tres tipos de focos ecogénicos para simplificar la observación, siendo el tipo 1 (FEP) el más característico de microcalcificaciones, el tipo 3 característico de coloide espeso o cristalizado, y el tipo 2 (FEI) como un grupo indeterminado sin cumplir criterios para definirlo como tipo 1 o 3 , en que la prudencia aconseja ser cauteloso en calificarlos como microcalcificaciones (Figura 11), ya que esto aumenta significativamente el puntaje en los sistemas de estratificación de riesgo usados actualmente (TIRADS, ATA) ${ }^{6-11}$, generando una PAF posiblemente innecesaria. Así, el objetivo es mejorar la especificidad en el diagnóstico de microcalcificaciones usando criterios más estrictos. En la práctica diaria es corriente ver en los informes ecográficos asumirse en forma indiscriminada los focos ecogénicos como microcalcificaciones, concordante con lo encontrado en el trabajo mencionado más arriba. El grado de sobrediagnóstico puede ser tal, que ciertos autores han llegado a proponer sólo usar el término "focos ecogénicos" de forma general sin diferenciar entre ellos ${ }^{13}$.

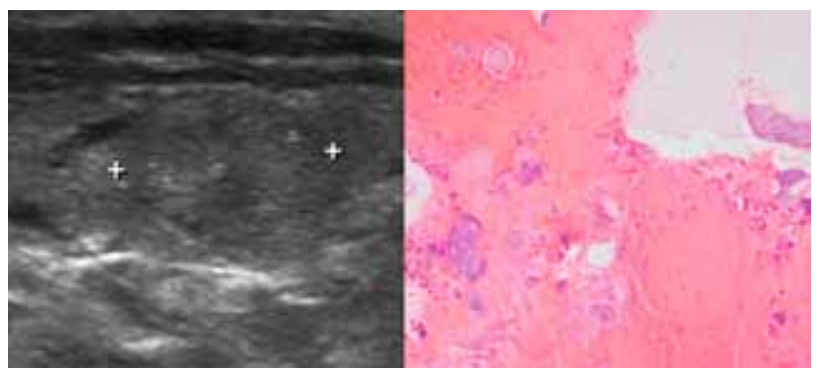

Figura 11. Hiperplasia folicular coloidea con focos ecogénicos informados erróneamente como microcalcificaciones. Biopsia descartó presencia de cuerpos de Psamomma y mostró focos de coloide espeso pequeños.

Literatura más reciente publicada por el comité de tiroides del Colegio Americano de Radiología en su consenso para la estratificación de riesgo de nódulos tiroideos (TI-RADS ACR) entrega información general para la asignación de puntaje en el score mencionado y se hace un intento por definir con mayor claridad a los FEP en relación a la presencia de CPs, mencionándolos como "más pequeños que macrocalcificaciones, sin sombra, y que si están presentes en el componente sólido del nódulo pudiesen corresponder a CPs" ${ }^{6}$. No obstante, nos parece una definición vaga, al estar basada sólo en opinión de expertos sin evidencia sólida fundamentada en estudios histológicos capaces de respaldar dicha afirmación, y que no entrega una solución efectiva al problema del sobrediagnóstico evidenciado por el estudio del año 2014 mencionado más arriba.

Con el fin de apoyar este planteamiento, se obtuvieron inicialmente los resultados mostrados en las tablas 2 y 3 , que evidenciaron una correlación moderadaalta entre $\mathrm{FEP} /$ malignidad y FEP/CPT, indicando un mejor rendimiento en comparación a los resultados de otros estudios. Se puede ver en la misma tabla que no hubo correlación significativa entre los otros focos ecogénicos y malignidad o CPT, favoreciendo el planteamiento que el hecho de identificar en ecografía correctamente las microcalcificaciones sí tiene una implicancia en el manejo de estos pacientes. Este último punto toma mucha más fuerza al observar lo que ocurre al mezclar FEP y FEI como si fueran una sola entidad (FEP+FEI en las tablas 2 y 3 ), viéndose que persiste una diferencia significativa con el grupo control en cuanto al riesgo de malignidad o CPT, sin embargo, se pierde totalmente el grado de asociación estadística que pudiese ser considerado significativo ( $V$ de Cramer $<0,3)$. Se evaluó también el valor del coloide como predictor de benignidad, sin embargo, no hubo asociación estadísticamente significativa $(p>0,05)$. Este último punto pudiese estar explicado por lo pequeño de dicho grupo $(5,71 \%$ del total de nódulos), aunque fue una proporción equivalente a lo reportado en otras series.

\section{Rendimiento de las definiciones propuestas y presencia de cuerpos de Psamomma}

Comparativamente entre el grupo general y sólo los CPT, hubo una diferencia marcada en la distribución de FEP vs FEI (Figura 6), apoyando que la definición planteada con el objetivo de mejorar la detección de CPs podría ser útil, considerando que éstos teóricamente debiesen presentarse casi exclusivamente en nódulos con $\mathrm{CPT}^{3}$.

En nuestro estudio encontramos que, de las 44 citologías, 5 (11,36\%) detectaron la presencia de CPs; de las 16 biopsias quirúrgicas disponibles para revisión de placas, $10(62,5 \%)$ mostraron CPs. Si bien en este contexto no se puede efectuar comparación alguna entre ambas modalidades de estudio, sí se muestra una tendencia a una mayor probabilidad de detectar CPs en la biopsia vs citología, lo que es concordante con resultados de trabajos previos. Cabe mencionar que fueron analizadas 4 biopsias de nódulos cuya ecografía informó ausencia de focos ecogénicos, que confirmaron la inexistencia de CPs (no se incluyó este dato en los resultados por su pequeño número). Al hacer la correlación inversa entre CPs en biopsia y aspecto ecográfico, hubo sólo un falso negativo, lo que confirma que la ecografía está expuesta a falsos negativos y que la detección de microcalcificaciones depende del número y ubicación, así como de la composición del nódulo.

Con respecto a lo definido por nuestro grupo 
como FEl, se observa una buena capacidad de este término para discriminar a los focos ecogénicos que no corresponden a microcalcificaciones, no existiendo en ninguno de ese grupo CPs en la histología. No obstante, desconocemos a qué corresponderían con exactitud. Entre las posibilidades, se encuentra que correspondan a paredes posteriores de microquistes, focos de coloide cuya cuantía no sea suficiente para generarse artefacto en cola de cometa $>1 \mathrm{~mm}$, focos fibróticos u otros. Aun así, en el contexto de los resultados obtenidos, esta diferenciación no parece tener relevancia ya que no se observa asociación significativa con malignidad o CPT en este estudio. Dado que en nuestras definiciones no incluimos el artefacto en cola de cometa $<1 \mathrm{~mm}$, y en consecuencia, aquellos fueron incluidos dentro de los FEl, pudiese ser que esté sobreestimado el indicador de asociación entre FEl y malignidad o CPT a pesar de su valor no significativo en nuestro análisis estadístico y, a su vez, que no hayamos sido capaces de aislar el posible valor de este tipo de focos descritos en la literatura como poseedores de una diferencia estadísticamente significativa con el grupo control en cuanto al riesgo de malignidad ${ }^{5,6}$. Esto último no nos parece una limitación importante del estudio dado que no estuvo dentro de los objetivos planteados inicialmente, además que se describe que su presencia, si bien sería significativa en cuanto al riesgo de malignidad, está en una baja proporción de nódulos tiroideos.

Para evaluar el valor clínico y diagnóstico de nuestra definición estricta de FEP en relación a CPs, se calcularon sensibilidad, especificidad y valores predictivos, con los resultados que se indican en la tabla 4. Como se puede ver, nuestra definición muestra un muy buen rendimiento para discriminar aquellos nódulos que tienen realmente CPs vs los que no tienen. Un aspecto claramente a mejorar es el VPP, dado que a pesar de haber hecho mucho más estrictos los criterios para informar FEP, se mantuvo un marcado sobrediagnóstico (13 verdaderos positivos vs 12 falsos positivos), aunque menor que en cualquier otro estudio disponible en la actualidad.

Se usó como gold standard de diagnóstico la cito-histología considerando la amplia evidencia existente en relación a la capacidad de citología y biopsia para el diagnóstico final en nódulos tiroideos ${ }^{15}$. Desafortunadamente, no se pudo tener acceso a las biopsias quirúrgicas de todos los pacientes con CPT por múltiples razones, observando en las 16 analizadas una tasa de detección mucho mayor que la tasa de detección de CPs de la citología pura. Considerando este último punto, se puede concluir que existe la posibilidad importante que la definición planteada tenga un VPP mucho mejor que el obtenido.

Mencionamos en los resultados la distribución respecto a la ecogenicidad y composición de los nódulos estudiados, dado que pudiesen haber sido estos factores contribuyentes a una mejor detección de FEP comparativamente con otras series en las que hay mayor proporción de nódulos con componente quístico $>50 \%$, así como mayor cantidad de nódulos hiperecogénicos. En nuestra serie, en el grupo de 44 CPT se obtuvieron: 2 nódulos hiperecogénicos $(4,5 \%)$; 12 isoecogénicos (27,3\%); 30 hipoecogénicos $(68,1 \%) ; 16$ nódulos predominantemente sólidos (36,4\%); 28 nódulos sólidos (63,6\%). Desconocemos si esta distribución es comparable o no con los CPT de distintos tamaños.

Nuestro estudio presenta múltiples e importantes limitaciones en el contexto de un diseño retrospectivo. Éstas incluyen la ausencia de estandarización en cuanto al tipo de haz de ultrasonido utilizado (multidireccional vs lineal), lo que puede determinar una diferencia importante en la detección de sombra acústica y discriminación de microquistes ${ }^{14}$, lo que a su vez pudiese ser la causa de generación del foco ecogénico, además del uso de equipos de dos fabricantes distintos. Los resultados fueron obtenidos sólo del grupo de nódulos con tamaño mayor a $3 \mathrm{~cm}$, lo que puede determinar un sesgo de selección importante. Asimismo, la determinación de malignidad/ benignidad estuvo mayormente basado en resultados de citologías sin confirmación con biopsia quirúrgica, aunque actualmente se considera la PAF como un examen de muy alta sensibilidad y especificidad, para categorías Bethesda $\mathrm{V}$ y $\mathrm{Vl}^{16}$. En cuanto a nuestras definiciones, a pesar de los buenos resultados obtenidos, éstas tienen un componente subjetivo no despreciable (problema inherente al examen dado su carácter operador-dependiente), lo que indica que el mejor rendimiento en comparación a otras series puede deberse de forma importante a que la determinación final de los focos ecogénicos se basó en el diagnóstico hecho por un operador experto, siendo posible que nuestros resultados no sean reproducibles por radiólogos con menos experiencia. Finalmente, la mayor de las grandes limitaciones de nuestro estudio fue la disponibilidad de sólo 16 placas de biopsias quirúrgicas para su revisión, habiendo un riesgo muy alto de sesgo al no haber sipo posible incluir 28 biopsias. Creemos que, si bien la citología ayudó a entregar 4 casos adicionales con CPs a los proporcionados por las biopsias analizadas, el estándar de oro real para la detección de CPs posiblemente se trate de la biopsia quirúrgica, por lo que el número total de pacientes con CPs podría encontrarse muy subestimado ${ }^{17}$.

\section{Conclusiones}

A pesar de las limitaciones de nuestro estudio, se observa una posibilidad de mejorar el problema del sobrediagnóstico de microcalcificaciones reales (CPs) al reformular la definición de microcalcificaciones con criterios más estrictos. En nuestra serie hay 
una buena correlación entre FEP y CPs, aunque el VPP resultante indica la necesidad de precisar aún mejor su aspecto.

Hasta el día de hoy no hay certeza en cuanto a la relevancia clínica real de hacer una diferenciación estricta de los múltiples focos ecogénicos encontrados en nódulos (excepto aquellos con artefacto en cola de cometa $>1 \mathrm{~mm}$ según lo sugerido en varias otras series). Sin embargo, creemos que esto se debe a la falta de una definición pictográfica precisa de estos distintos elementos y que lograr aislar el aspecto de las microcalcificaciones sí es relevante, como indican nuestros resultados y algunos estudios histológicos. Así, queda en manifiesto la necesidad de diseñar e implementar estudios prospectivos que prueben con solidez nuestras hipótesis, con la intención de llegar a una modificación del paradigma actual.

\section{Referencias}

1. Takashima S, Fukuda $\mathrm{H}$, Nomura $\mathrm{N}$, Kishimoto $\mathrm{H}$, Kim T, Kobayashi T. Thyroid nodules: Re-evaluation with ultrasound. Journal of Clinical Ultrasound 1995; 23(3): 179-184.

2. Khoo M, Asa S, Witterick I, Freeman J. Thyroid calcification and its association with thyroid carcinoma. Head \& Neck 2002; 24(7): 651-655.

3. Triggiani V, Guastamacchia E, Licchelli B, Tafaro E. Microcalcifications and Psammoma Bodies in Thyroid Tumors. Thyroid 2008; 18(9): 1017-1018.

4. Das D. Psammoma body: A product of dystrophic calcification or of a biologically active process that aims at limiting the growth and spread of tumor? Diagnostic Cytopathology 2009; 37(7): 534-541.

5. Malhi H, Beland M, Cen S, Allgood E, Daley K, Martin S, et al. Echogenic Foci in Thyroid Nodules: Significance of Posterior Acoustic Artifacts. American Journal of Roentgenology 2014; 203(6): 1310-1316.

6. Tessler F, Middleton W, Grant E, Hoang J, Berland L, Teefey S, et al. ACR Thyroid Imaging, Reporting and Data System (TI-RADS): White Paper of the ACR TIRADS Committee. Journal of the American College of Radiology 2017; 14(5): 587-595.

7. Hoang J, Lee W, Lee M, Johnson D, Farrell S. US Features of Thyroid Malignancy: Pearls and Pitfalls. RadioGraphics 2007; 27(3): 847-860.
8. Horvath E, Majlis S, Rossi R, Franco C, Niedmann J, Castro A, et al. An Ultrasonogram Reporting System for Thyroid Nodules Stratifying Cancer Risk for Clinical Management. The Journal of Clinical Endocrinology \& Metabolism 2009; 94(5): 1748-1751.

9. Russ G. Risk stratification of thyroid nodules on ultrasonography with the French TI-RADS: description and reflections. Ultrasonography 2016; 35(1): 25-38.

10. Shin J, Baek J, Chung J, Ha E, Kim J, Lee Y, et al. Ultrasonography Diagnosis and Imaging-Based Management of Thyroid Nodules: Revised Korean Society of Thyroid Radiology Consensus Statement and Recommendations. Korean Journal of Radiology 2016; 17(3): 370.

11. Haugen B, Alexander E, Bible K, Doherty G, Mandel S, Nikiforov Y, et al. 2015 American Thyroid Association Management Guidelines for Adult Patients with Thyroid Nodules and Differentiated Thyroid Cancer: The American Thyroid Association Guidelines Task Force on Thyroid Nodules and Differentiated Thyroid Cancer. Thyroid 2016; 26(1): 1-133.

12. Mosso L, Campusano C, González H, Domínguez J, Salman P, Suazo V, et al. Del macro al microcarcinoma tiroideo: cambios en las características de presentación del cáncer de tiroides en un centro universitario chileno en 20 años. Rev Méd Chile 2013; 141(4): 442-448.

13. Yoon J, Kim E, Son E, Moon H, Kwak J. Diffuse Microcalcifications Only of the Thyroid Gland Seen on Ultrasound: Clinical Implication and Diagnostic Approach. Annals of Surgical Oncology 2011; 18(10): 2899-2906.

14. Muradali D, Colgan T, Hayeems E, Burns P, Wilson S. Echogenic Ovarian Foci without Shadowing: Are They Caused by Psammomatous Calcifications? Radiology 2002; 224(2): 429-435.

15. Pinchot S. Accuracy of Fine-Needle Aspiration Biopsy for Predicting Neoplasm or Carcinoma in Thyroid Nodules $4 \mathrm{~cm}$ or Larger. Archives of Surgery 2009; 144(7): 649.

16. Bongiovanni M, Spitale A, Faquin W, Mazzucchelli L, Baloch Z. The Bethesda System for Reporting Thyroid Cytopathology: A Meta-Analysis. Acta Cytologica 2012; 56(4): 333-339.

17. Ellison E, Lapuerta P, Martin S. Psammoma bodies in fine-needle aspirates of the thyroid. Cancer 1998; 84(3): 169-175. 